\title{
Element-sensitive measurement of the hole-nuclear spin interaction in quantum dots
}

\author{
E. A. Chekhovich ${ }^{1 \star}$, M. M. Glazov ${ }^{2,3}$, A. B. Krysa ${ }^{4}$, M. Hopkinson ${ }^{4}$, P. Senellart ${ }^{5}$, A. Lemaître ${ }^{5}$, \\ M. S. Skolnick ${ }^{1}$ and A. I. Tartakovskii ${ }^{1 \star}$
}

It has been proposed that valence-band holes can form robust spin qubits ${ }^{1-4}$ owing to their weaker hyperfine coupling compared with electrons ${ }^{5,6}$. However, it was demonstrated recently $^{7-11}$ that the hole hyperfine interaction is not negligible, although a consistent picture of the mechanism controlling its magnitude is still lacking. Here we address this problem by measuring the hole hyperfine constant independently for each chemical element in InGaAs/GaAs, InP/GalnP and GaAs/AIGaAs quantum dots. Contrary to existing models ${ }^{10,11}$ we find that the hole hyperfine constant has opposite signs for cations and anions and ranges from $-15 \%$ to $+15 \%$ relative to that for electrons. We attribute such changes to the competing positive contributions of $p$-symmetry atomic orbitals and the negative contributions of $d$-orbitals. These findings yield information on the orbital composition of the valence band ${ }^{12}$ and enable a fundamentally new approach for verification of computed Bloch wavefunctions in semiconductor nanostructures ${ }^{13}$. Furthermore, we show that the contribution of cationic $d$-orbitals leads to a new mechanism of hole spin decoherence.

Owing to the s-type character of the Bloch wavefunction, the hyperfine interaction of the conduction band electrons is isotropic (the Fermi contact interaction) and is described by a single hyperfine constant $A$, positive $(A>0)$ for most III-V semiconductors and proportional to the electron density at the nucleus. In contrast, for valence-band holes the contact interaction vanishes owing to the symmetry properties of the wavefunction, and the non-local dipole-dipole interaction dominates ${ }^{10,11,13-15}$. As a result, the sign, magnitude and anisotropy of the hyperfine interaction depend on the actual form of the valence-band Bloch wavefunction, which is usually not available with sufficient precision. Thus, predicting the properties of the hole hyperfine coupling using first-principle calculations remains a difficult task.

In this work we perform direct measurements of the hyperfine constants that describe the hole hyperfine interaction with nuclear spins polarized along the growth axis of the structure (that is, the diagonal elements of the hole hyperfine Hamiltonian). This is achieved by simultaneous and independent detection of the electron and hole Overhauser shifts using high-resolution photoluminescence spectroscopy of neutral quantum dots. In contrast to previous work $^{9}$, we now also apply excitation with a radiofrequency oscillating magnetic field, which allows isotopeselective probing of the valence-band hole hyperfine interaction ${ }^{16}$. Using this technique we find that in all studied materials, cations (gallium, indium) have a negative hole hyperfine constant, whereas it is positive for anions (phosphorus, arsenic), a result attributed to the previously disregarded contribution of the cationic $d$-shells into the valence-band Bloch wavefunctions.

Using the experimentally measured diagonal components of the hyperfine Hamiltonian (hole hyperfine constants) we calculate its non-diagonal part. We show that the admixture of the $d$-shells has a major effect on the symmetry of the hyperfine Hamiltonian in quantum dots: unlike pure heavy holes constructed only of $p$-symmetry shells for which the hyperfine interaction has an Ising form ${ }^{11}$, the $d$-shell contribution results in non-zero non-diagonal elements of the hyperfine Hamiltonian. We predict this to be a major source of heavy-hole spin decoherence.

Our experiments rely on detection of photoluminescence of both bright and dark neutral excitons $s^{9,17-19}$ formed by electrons $\uparrow(\downarrow)$ with spin $\pm 1 / 2$ and heavy holes $\Uparrow(\Downarrow)$ with momentum $\pm 3 / 2$ parallel (antiparallel) to the growth axis $\mathrm{Oz}$ (Fig. 1a). As the quantum dots contain of the order of $10^{5}$ nuclei, non-zero average nuclear spin polarization of the $k$ th isotope $\left\langle I_{z}^{k}\right\rangle$ along the $O z$ axis can be treated as an additional magnetic field acting on the electron and hole spins. The coupling strength of the electron to the nuclear spins of isotope $k$ is described by the hyperfine constant $A^{k}$. The additional energy of the exciton state with electron spin $\uparrow(\downarrow)$ is equal to $+(1 / 2) \Delta E_{\mathrm{e}}^{k}\left(-(1 / 2) \Delta E_{\mathrm{e}}^{k}\right)$, where the electron hyperfine shift induced by the $k$ th isotope is defined as

$$
\Delta E_{\mathrm{e}}^{k}=\rho^{k} A^{k}\left\langle I_{z}^{k}\right\rangle
$$

with $\rho^{k}$ describing the relative concentration of the $k$ th isotope. For the heavy-hole states the hyperfine interaction is described using a constant $C^{k}$ expressed in terms of the normalized heavy-hole hyperfine constant $\gamma^{k}$ as $C^{k}=\gamma^{k} A^{k}$. The variation of the energy of the exciton with hole spin $\Uparrow(\Downarrow)$ is $+(1 / 2) \Delta E_{\mathrm{h}}^{k}\left(-(1 / 2) \Delta E_{\mathrm{h}}^{k}\right)$, where the hole hyperfine shift is

$$
\Delta E_{\mathrm{h}}^{k}=\rho^{k} \gamma^{k} A^{k}\left\langle I_{z}^{k}\right\rangle
$$

By taking the same values of $\rho^{k}$ in equations (1) and (2) we assume for simplicity a uniform distribution of the average nuclear spin polarization and concentration of chemical elements within the volume of the quantum dot. According to equations (1) and (2), hole and electron hyperfine shifts depend linearly on each other $\left(\Delta E_{\mathrm{h}}^{k}=\gamma^{k} \Delta E_{\mathrm{e}}^{k}\right)$. In the experiment, hyperfine shifts are measured

\footnotetext{
${ }^{1}$ Department of Physics and Astronomy, University of Sheffield, Sheffield S3 7RH, UK, ${ }^{2}$ loffe Physical-Technical Institute of RAS, St Petersburg 194021 Russia, ${ }^{3}$ Spin Optics Laboratory, St Petersburg State University, St Petersburg 198504, Russia, ${ }^{4}$ Department of Electronic and Electrical Engineering, University of Sheffield, Sheffield S1 3JD, UK, ${ }^{5}$ Laboratoire de Photonique et de Nanostructures, Route de Nozay, 91460 Marcoussis, France. *e-mail: e.chekhovich@sheffield.ac.uk; a.tartakovskii@sheffield.ac.uk.
} 

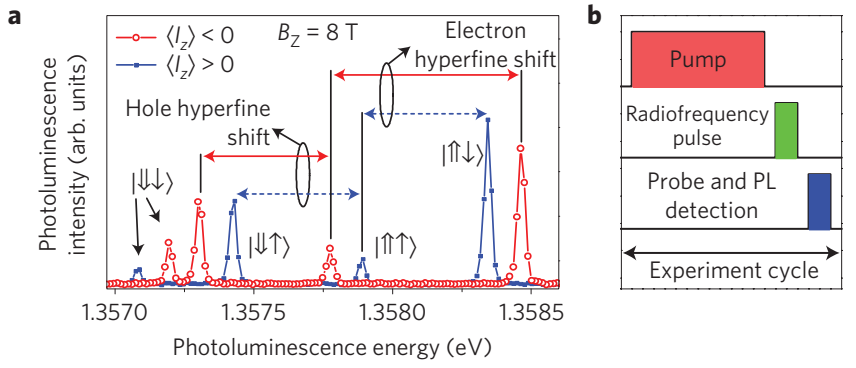

Figure 1 | Optical techniques for isotope-selective measurement of the hole hyperfine constants. a, Photoluminescence spectra of a single neutral $\operatorname{lnGaAs} / \mathrm{GaAs}$ quantum dot in a magnetic field $B_{\mathrm{z}} \approx 8.0 \mathrm{~T}$. For low-power optical excitation, four photoluminescence lines are observed in each spectrum corresponding to all possible combinations of the electron spin states $(\uparrow, \downarrow)$ and hole states $(\Uparrow, \downarrow)$ forming two bright excitons $(|\Uparrow \downarrow\rangle$, $|\Downarrow \uparrow\rangle)$ and two dark excitons $(|\Uparrow \uparrow\rangle,|\Downarrow \downarrow\rangle)$ that have a small $(<0.01)$ admixture of bright states making dark states visible in photoluminescence spectra $^{9,17}$. To demonstrate independent detection of electron and hole hyperfine shifts two spectra are shown corresponding to negative (open symbols) and positive (solid symbols) nuclear spin polarization $\left\langle I_{z}\right\rangle$ induced on the dot by pumping with $\sigma^{+}$and $\sigma^{-}$polarized light, respectively. b. Timing diagram of the pump-probe experiment used in the measurements of the hole hyperfine constants: nuclear spins are polarized by a high-power optical pump pulse. Following this, a radiofrequency oscillating magnetic field is switched on to achieve isotope-selective depolarization of nuclear spins. Finally, the sample is excited with a low-power probe laser pulse, during which the photoluminescence spectrum of both bright and dark excitons (similar to that in $\mathbf{a}$ ) is measured. See further details of experimental techniques in Methods.

from photoluminescence spectra of the quantum dot (see Fig. 1a) and the slope of the resulting dependence of $\Delta E_{\mathrm{h}}^{k}$ on $\Delta E_{\mathrm{e}}^{k}$ is used to determine $\gamma^{k}$ (see further details in Methods).

We start by presenting results for strain-free GaAs/AlGaAs quantum dots. The dependence of $\Delta E_{\mathrm{h}}^{k}$ on $\Delta E_{\mathrm{e}}^{k}$ for $k=$ As (squares) and $k=\mathrm{Ga}$ (circles) is shown in Fig. 2a for GaAs quantum dot A1. It can be seen that the dependences for both Ga and As are linear as predicted by equations (1) and (2). Fitting gives the following values for the hole hyperfine constants $\gamma^{\mathrm{Ga}}=-7.0 \pm 4.0 \%$ and $\gamma^{\mathrm{As}}=+15.0 \pm 4.5 \%$. Similar measurements were performed on three other GaAs quantum dots. The resulting values are given in Table 1. As the variation between different dots is within experimental error, we take average values for all dots yielding $\gamma^{\mathrm{Ga}}=-7.5 \pm 3.0 \%$ and $\gamma^{\mathrm{As}}=+16.0 \pm 3.5 \%$. We thus conclude that different chemical elements have opposite signs of the hole hyperfine constants: they are positive for arsenic and negative for gallium. This is an unexpected result in comparison with previous theoretical studies ${ }^{10,11}$ and experiments insensitive to individual chemical elements where negative values of $\gamma$ have been found in InP and InGaAs quantum $\operatorname{dots}^{8,9}$.

We have also performed isotope-sensitive measurements of the hole nuclear interaction in InGaAs/GaAs quantum dots. The dependence of $\Delta E_{\mathrm{h}}^{k}$ on $\Delta E_{\mathrm{e}}^{k}$ for InGaAs quantum dot B1 is shown in Fig. $2 \mathrm{~b}$ for ${ }^{71} \mathrm{Ga}$ (circles), ${ }^{75} \mathrm{As}$ (squares) and for the total hyperfine shifts of ${ }^{69} \mathrm{Ga}$ and ${ }^{115} \mathrm{In}$ (triangles). The values of $\gamma^{k}$ obtained from the fitting (see details in Methods) are summarized in Table 1. Similar to GaAs, we find that arsenic has a positive hole hyperfine constant whereas for gallium and indium it is negative.

Applying the isotope selection techniques to InP/GaInP quantum dots studied previously ${ }^{9}$ we find $\gamma^{\text {In }}=-12.5 \pm 3.0 \%$, consistent with our previous results obtained without isotope selection'. Similar to GaAs and InGaAs quantum dots, we find a large positive constant for anions (phosphorus) $\gamma^{\mathrm{P}}=+18.0 \pm 8.0 \%$.
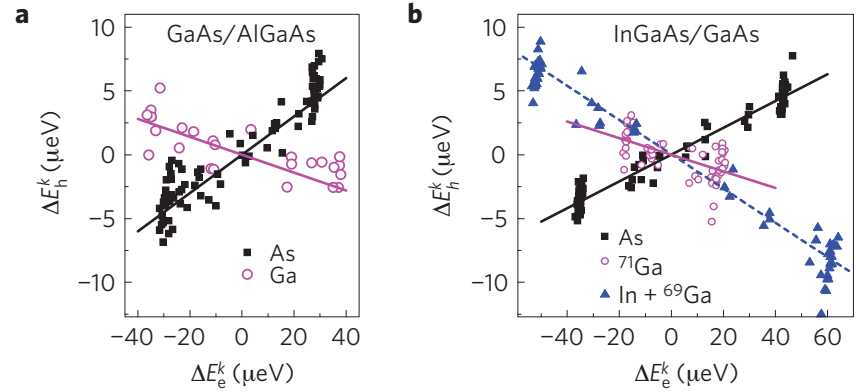

Figure 2 | Experimental results of the isotope-selective hole hyperfine measurements. $\mathbf{a}, \mathbf{b}$, Dependence of the hole hyperfine shift $\Delta E_{\mathrm{h}}^{k}$ on the electron hyperfine shift $\Delta E_{\mathrm{e}}^{k}$ for different isotopes in GaAs quantum dot $\mathrm{A} 1$ (a) and InGaAs quantum dot B1 (b). Solid lines show fitting: the slopes correspond to the relative hole-nuclear hyperfine constants $\gamma^{k}$ (see details in Methods). We find $\gamma^{\mathrm{Ga}} \approx-7.0 \%, \gamma^{\mathrm{As}} \approx+15.0 \%$ for GaAs quantum dot $\mathrm{A} 1$ and $\gamma^{\mathrm{Ga}} \approx-6.5 \%, \gamma^{\mathrm{As}} \approx+10.5 \%$ for InGaAs quantum dot B1. As the NMR resonances of ${ }^{69} \mathrm{Ga}$ and ${ }^{115} \mathrm{In}$ in In GaAs cannot be resolved ${ }^{16}$, we measure the total hyperfine shifts $\Delta E_{\mathrm{e}}^{\ln +{ }^{69} \mathrm{Ga}}$ and $\Delta E_{\mathrm{h}}^{\mathrm{ln}+69} \mathrm{Ga}$ produced by these isotopes. Further analysis gives $\gamma^{\ln } \approx-16.0 \%$ for quantum dot $B 1$ (see Supplementary Section S3B). The dashed line in $\mathbf{b}$ is a guide to the eye.

The values of $\gamma$ presented in Table 1 describe the hyperfine interaction of the valence-band states that are in general mixed states of heavy and light holes. However, as we show in detail in Supplementary Section S5, such mixing cannot account for the opposite signs of $\gamma^{k}$ observed for the cations and anions, but might be the reason for the dot-to-dot variation of $\gamma^{\text {In }}$ observed in InP quantum dots (see Table 1). Such variation may also arise from the dot-to-dot-dependent spatial separation of electron and hole wavefunctions ${ }^{8}$.

From the measurements without radiofrequency pulses (similar to earlier isotope-non-selective experiments of refs 8,9), we find that in GaAs quantum dots the total hole hyperfine shift (induced by all isotopes) is positive and amounts to $\gamma \approx+5 \%$ relative to the total electron hyperfine shift. For the studied InGaAs quantum dots where indium and gallium concentrations are estimated to be $\rho^{\mathrm{In}} \approx 20 \%$ and $\rho^{\mathrm{Ga}} \approx 80 \%$ (see ref. 16), we find negative $\gamma \approx-4 \%$, whereas for more indium-rich InGaAs dots emitting at $E_{\mathrm{PL}} \sim 1.30 \mathrm{eV}$ the value of $\gamma \approx-9 \%$ has been reported ${ }^{8}$. This suggests that for quantum dots with a particular indium concentration $\left(\rho^{\mathrm{In}} \sim 10 \%\right)$ one can expect close to zero $(\gamma \approx 0)$ total hole hyperfine shift induced by nuclear spin polarization along the $\mathrm{Oz}$ direction. Hole spin qubits in such structures will be insensitive to static nuclear fields that are induced by the optical control pulses and cause angle errors in spin rotations. Such spin qubits will benefit from a simplified implementation of the coherent control protocols 4 .

We now turn to analysis of the experimental results presented in Table 1. First-principle calculation of the valence-band hyperfine coupling requires integration of the hyperfine Hamiltonian using explicit expressions for the Bloch wavefunctions. However, it has been shown that reasonable estimates can be obtained using a simplified approach ${ }^{10,11,15}$ : Bloch wavefunctions can be approximated by linear combinations of hydrogenic wavefunctions (further details may be found in Methods). Previous calculations ${ }^{10,11,15}$ based on this approach considered valence-band states constructed from atomic $p$-orbitals (with orbital momentum $l=1$ ), which yields positive hole hyperfine constant $\gamma^{k}>0$ for all chemical elements, in contradiction with our experimental findings.

This disagreement can be overcome by taking into account the contribution of shells with higher orbital momenta $l$, resulting in more accurate approximation of the hole wavefunction ${ }^{20}$ : in particular we consider the contribution of the $d$-shell states 
Table 1 | Experimentally measured photoluminescence energies $E_{\mathrm{PL}}$ and hole hyperfine constants $\gamma^{k}$ for different chemical elements $k$ in several GaAs and InGaAs quantum dots.

\begin{tabular}{|c|c|c|c|c|c|}
\hline Material/quantum dot & $\gamma^{\mathrm{Ga}}(\%)$ & $\gamma^{\ln }(\%)$ & $\gamma^{\text {As }}(\%)$ & $\gamma^{\mathrm{P}}(\%)$ & $E_{\mathrm{PL}}(\mathrm{eV})$ \\
\hline \multicolumn{6}{|c|}{ GaAs/AIGaAs } \\
\hline QD A1 & $-7.0 \pm 4.0$ & - & $+15.0 \pm 4.5$ & - & 1.713 \\
\hline QD A2 & $-8.5 \pm 3.5$ & - & $+17.0 \pm 5.0$ & - & 1.713 \\
\hline QD A3 & $-5.5 \pm 4.5$ & - & $+15.0 \pm 4.0$ & - & 1.702 \\
\hline QD A4 & $-7.5 \pm 4.5$ & - & $+18.5 \pm 5.5$ & - & 1.707 \\
\hline \multicolumn{6}{|c|}{$\operatorname{lnGaAs} / \mathrm{GaAs}$} \\
\hline QD B1 & $-6.5 \pm 5.5$ & $-16.0 \pm 4.0$ & $+10.5 \pm 2.0$ & - & 1.358 \\
\hline QD B2 & $-3.0 \pm 6.5$ & $-15.5 \pm 5.0$ & $+10.0 \pm 3.0$ & - & 1.358 \\
\hline QD B3 & $-5.5 \pm 5.0$ & $-16.0 \pm 4.0$ & $+8.0 \pm 2.0$ & - & 1.357 \\
\hline QD B4 & $-4.5 \pm 7.0$ & $-13.0 \pm 4.5$ & $+8.5 \pm 3.0$ & - & 1.357 \\
\hline \multicolumn{6}{|c|}{$\ln \mathrm{P} / \mathrm{G} a \ln \mathrm{P}$} \\
\hline QD C1 & - & $-15.5 \pm 1.5$ & - & $+17.5 \pm 11.0$ & 1.834 \\
\hline QD C2 & - & $-15.0 \pm 1.5$ & - & $+18.5 \pm 12.5$ & 1.851 \\
\hline QD C3 & - & $-9.0 \pm 1.5$ & - & $+19.0 \pm 12.0$ & 1.844 \\
\hline QD C4 & - & $-10.5 \pm 1.5$ & - & $+17.5 \pm 11.0$ & 1.834 \\
\hline \multicolumn{6}{|c|}{ Average } \\
\hline $\mathrm{GaAs} / \mathrm{AlGaAs}$ & $-7.5 \pm 3.0$ & - & $+16.0 \pm 3.5$ & - & \\
\hline $\ln G a A s / G a A s$ & $-5.0 \pm 4.5$ & $-15.0 \pm 3.5$ & $+9.0 \pm 2.0$ & - & \\
\hline $\ln \mathrm{P} / \mathrm{G} a \ln \mathrm{P}$ & - & $-12.5 \pm 3.0$ & - & $+18.0 \pm 8.0$ & \\
\hline
\end{tabular}

Error estimates give $90 \%$ confidence values. Average values for each chemical element in each material are given at the bottom of the table.

$(l=2)$. Both $p$ - and $d$-orbitals are schematically depicted in Fig. 3a. We assume that the heavy-hole states can be taken as linear combinations of $p$-shells with weight $\alpha_{p}$ and $d$-shells with weight $\alpha_{d}\left(\left|\alpha_{p}\right|^{2}+\left|\alpha_{d}\right|^{2}=1\right)$. Calculation of the relative hole hyperfine constant yields:

$$
\begin{gathered}
\gamma^{k}=\frac{12}{5}\left|\alpha_{p}\right|^{2} M_{p}-\frac{18}{7}\left|\alpha_{d}\right|^{2} M_{d} \\
M_{l}=\frac{1}{|S(0)|^{2}} \int_{0}^{\infty} \frac{R_{l}^{2}(r)}{r} \mathrm{~d} r
\end{gathered}
$$

where positive integrals $M_{l}(l=p, d)$ depend on the hydrogenic radial wavefunctions $R_{l}(r)$ corresponding to the shell with orbital momentum $l$ and normalized by the density $(4 \pi)^{-1}|S(0)|^{2}$ of the conduction band electron wavefunction at the nuclear site (see further information in Methods and Supplementary Section S4).

It follows from equation (3) that unlike the $p$-shell, the $d$-shell gives rise to a negative contribution to $\gamma^{k}$ : importantly the sign of the hyperfine interaction is totally determined by the angular symmetry of the wavefunction, whereas the radial part $R_{l}(r)$ affects only the magnitude of the contribution. We note that any hybridization of the valence-band states with s-orbitals due to quantum-dot symmetry reduction would lead to a positive contribution to $\gamma^{k}$ and thus cannot account for the negative hyperfine constants ${ }^{11}$. To obtain numerical estimates we consider GaAs material and approximate $S(r), \mathrm{R}_{p}(r), \mathrm{R}_{d}(r)$ with radial hydrogenic wavefunctions corresponding to $4 s-, 4 p$ - and $3 d$-shells, respectively, taken with effective orbital radii ${ }^{11,21}$. The resulting calculated dependence of $\gamma^{k}$ on $d$-shell admixture $\left|\alpha_{d}\right|^{2}$ is shown in Fig. $3 \mathrm{~b}$ for $k=\mathrm{Ga}$ and $k=$ As nuclei. On comparing this with the experimental results of Table 1 (shown by the horizontal bands in Fig. 3b), we conclude that the symmetry of the wavefunction at the anions (arsenic) is close to pure $p$-type, whereas for the cation gallium a significant contribution of the $d$-shell $(\sim 20 \%)$ is required to account for the negative hole hyperfine constant measured experimentally.

The non-zero contribution of the $d$-symmetry orbitals has a further unexpected effect on the hole hyperfine interaction: we find (see Supplementary Section S5) that the hyperfine interaction induces spin flips between the heavy-hole states $\Uparrow$ and $\Downarrow$. This is in contrast to the case of pure heavy holes constructed only of $p$-symmetry states for which the hyperfine interaction has an Ising form ${ }^{11}$ : in that case the symmetry of the system is artificially raised to spherical, resulting in hyperfine interaction conserving angular momenta. The inclusion of the $d$-shells reduces the symmetry of the system down to that of the real crystal (described by the $T_{d}$ point group). Under these conditions the hyperfine interaction does not conserve angular momentum and has non-zero non-diagonal elements coupling heavy holes with the opposite spins.

It was demonstrated previously that heavy-light hole mixing can result in a non-Ising form of the hyperfine interaction ${ }^{10,11}$. However, our estimates show that for gallium in GaAs the contribution of the $d$-shells to the non-diagonal matrix elements of the hyperfine Hamiltonian dominates over the effect of the heavy-light hole mixing even if the valence-band states have a light-hole contribution as large as $\sim 30 \%$ (see Supplementary Section S5). A similar effect is expected for the other materials studied, because for all of them significant contribution of the cation $d$-shells is observed (resulting in $\gamma^{k}<0$ ). Thus, the $d$-orbital contribution will be a source of heavy-hole spin dephasing even in the absence of mixing with light holes and should be taken into account when analysing experimentally measured hole spin coherence times.

The hyperfine interaction is particularly strong in the small volume around the atomic core ${ }^{13}$. To estimate this volume we limit the integration in equation (3) to a sphere of a radius $r_{0}$, which makes $M_{l}$ (and hence $\gamma^{k}$ ) a function of $r_{0}$. The 
a

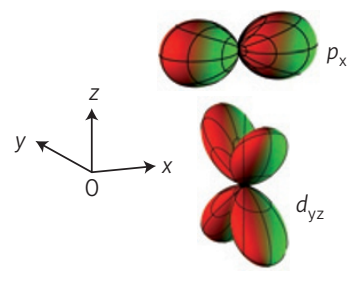

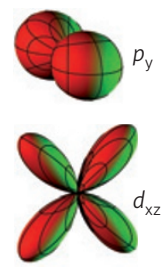

C b

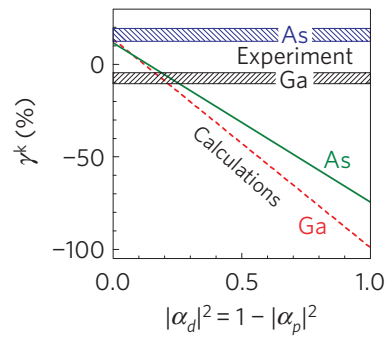

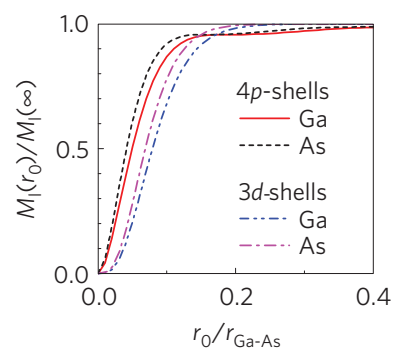

Figure 3 | Model calculations of the hole hyperfine constants.

a, Schematic representations of $p$ - and $d$-orbitals that transform according to the $F_{2}$ representation of the $T_{d}$ point group of the crystal symmetry.

b, Calculated dependence of the relative hole hyperfine constant $\gamma^{k}$ of $\mathrm{Ga}$ and $A s$ as a function of $d$-shell contribution $\left|\alpha_{d}\right|^{2}$ (lines). Horizontal bands show experimentally measured confidence intervals for $\gamma^{k}$ for GaAs quantum dots (see Table 1). c, Dependence of integrals $M_{l}\left(r_{0}\right)$ (see equation (3)) on the upper integration limit $r_{0}$ for $3 d$ - and $4 p$-shells for both $G$ a and As. Values of $M_{l}\left(r_{0}\right)$ are normalized by their values at $r_{0} \rightarrow \infty$, and $r_{0}$ is normalized by the distance between $\mathrm{Ga}$ and As nuclei $r_{\mathrm{Ga}-\mathrm{As}} \approx 0.245 \mathrm{~nm}$ in $\mathrm{GaAs}$. The rapid saturation of the $M_{l}\left(r_{0}\right)$ variations shows that the major contribution to the hole hyperfine interaction $(>95 \%)$ arises from a small volume with a radius of $r_{0} \lesssim 0.15 \times r_{G a-A s}$ around the nucleus.

dependence of $M_{l}\left(r_{0} / r_{\mathrm{Ga}-\mathrm{As}}\right)$ on the radius of the integration sphere, normalized by the distance between nearest $\mathrm{Ga}$ and As neighbours $r_{\mathrm{Ga}-\mathrm{As}} \approx 0.245 \mathrm{~nm}$, is shown in Fig. $3 \mathrm{c}$ for $3 d$ - and $4 p$-shells for both Ga and As (because $M_{l}\left(r_{0} \rightarrow \infty\right)$ converges it is normalized by its limiting value $\left.M_{l}(\infty)\right)$. It can be seen that the main contribution to the integral $(>95 \%)$ comes from the small volume within a sphere with a radius of $\sim 0.15 \times r_{\mathrm{Ga}-\mathrm{As}}$, whereas the outer volume gives only a minor contribution owing to the rapid decrease of the dipole-dipole interaction strength with increasing distance. Thus, hyperfine coupling can be used to probe the structure of the wavefunction in the atomic core.

Our results on Bloch wavefunction orbital composition are in general agreement with existing theoretical models: the importance of $d$-shells in describing the valence-band states is well recognized $^{20,22}$, and it has been shown that the $d$-symmetry contribution originates mainly from cations. However, the previous reports $^{12,23,24}$ predicted a much larger $d$-symmetry contribution $\left(\left|\alpha_{d}\right|^{2}\right.$ exceeding 50\%) than estimated in our work $\left(\left|\alpha_{d}\right|^{2} \sim 20 \%\right)$. Such deviation might be due to the simplified character of our calculations and/or due to the intrinsic limitations of the wavefunction modelling techniques such as tight-binding or pseudo-potential methods ${ }^{25}$ that fail to reproduce the wavefunction structure in the vicinity of the nucleus.

Theoretical modelling of the microscopic wavefunctions allows band structures to be calculated and thus is of importance both for fundamental studies and technological applications of semiconductors ${ }^{25}$. However, as true first-principle calculation of the many-body wavefunction is highly challenging, empirical approaches are normally used. They ultimately rely on fitting model parameters to describe the set of experimental data (for example, energy gaps, effective masses, X-ray photoemission spectra). The experimental data on the valence-band hyperfine parameters obtained in this work provide a means for probing the hole Bloch wavefunction: they allow direct analysis of the wavefunction orbital composition in the close vicinity of the nuclei, where theoretical modelling is the most difficult. Furthermore, our experimental method is unique in being isotopeselective, thus allowing independent study of cation and anion wavefunctions. The techniques developed in this work for quantum dots have the potential to be extended to other semiconductor systems, for example, bound excitons in III-V and group-IV bulk semiconductors where dark excitons are observed ${ }^{26}$ and hyperfine shifts can be induced and detected ${ }^{27}$.

A rigorous modelling of the hyperfine parameters $^{13}$ has not been carried out so far for the valence-bands states of III-V semiconductor nanostructures. Progress in this direction will provide a better understanding of the mechanisms controlling the sign and magnitude of the valence-band hyperfine coupling. In particular, the potential effect of large inhomogeneous elastic strain (present in self-assembled quantum dots) on the microscopic Bloch hole wavefunction needs to be examined. This may be a possible route to engineering of holes with reduced hyperfine coupling.

\section{Methods}

Samples and experimental techniques. Our experiments were performed on undoped GaAs/AlGaAs (ref. 28), InP/GaInP (ref. 9) and InGaAs/GaAs (ref. 16) quantum-dot samples without electric gates (further details can be found in Supplementary Section S1). The photoluminescence spectra of neutral quantum dots placed at $T=4.2 \mathrm{~K}$, in an external magnetic field $B_{z}$ normal to the sample surface, were measured using a double spectrometer and a CCD (charge-coupled device) camera.

Detection of the hyperfine shifts, required to measure hole hyperfine constants is achieved using pump-probe techniques ${ }^{9}$ (see timing diagram in Fig. 1b). Nuclear spin polarization is prepared with a long $(\sim 6 \mathrm{~s})$ high-power optical pump pulse. Following this, a radiofrequency oscillating magnetic field is switched on to achieve isotope-selective depolarization of nuclear spins (radiofrequency pulse duration varies between 0.15 and $35 \mathrm{~s}$ depending on the material). Finally, the sample is excited with a low-power short $(\sim 0.3 \mathrm{~s})$ probe laser pulse, during which the photoluminescence spectrum (see Fig. 1a) of both bright and dark excitons is measured. In all experiments the durations of the radiofrequency and probe pulses are much smaller than the natural decay time of the nuclear polarization. See further details of experimental techniques in Supplementary Sections S2 and S3.

Techniques for isotope-selective measurement of the hole hyperfine constant. The concept of the valence-band hyperfine constant measurement is based on detecting hole hyperfine shift $\Delta E_{\mathrm{b}}^{k}$ (equation (2)) as a function of electron hyperfine shift $\Delta E_{\mathrm{e}}^{k}$ (equation (1)) by varying the nuclear spin polarization $\left\langle I_{z}^{k}\right\rangle$. Non-zero $\left\langle I_{z}^{k}\right\rangle$ is induced by optical nuclear spin pumping: circularly polarized light of the pump laser generates spin-polarized electrons that transfer their polarization to nuclei ${ }^{16,17,29}$ through the hyperfine interaction. The magnitude of $\left\langle I_{z}^{k}\right\rangle$ is controlled by changing the degree of circular polarization ${ }^{9}$. According to equations (1) and (2), hole and electron hyperfine shifts depend linearly on each other $\left(\Delta E_{\mathrm{h}}^{k}=\gamma^{k} \Delta E_{\mathrm{e}}^{k}\right)$ with a slope equal to the normalized hole hyperfine constant $\gamma^{k}$. The electron (hole) hyperfine shift of a chosen $(k$ th) isotope is deduced from a differential measurement: the spectral splitting between excitons with opposite electron (hole) spins (Fig. la) is measured with a radiofrequency pulse that depolarizes only the $k$ th isotope and without any radiofrequency pulse. The difference between these two splittings is equal to $\Delta E_{\mathrm{e}}^{k}\left(\Delta E_{\mathrm{b}}^{k}\right)$. As an example, Fig. 2 shows measurements where electron hyperfine shift $\Delta E_{\mathrm{e}}^{k}$ for isotope $k$ is found as the difference of the energy splitting $\left(E_{\Uparrow \uparrow}-E_{\Uparrow \downarrow}\right)$ of the $\Uparrow \uparrow$ and $\Uparrow \downarrow$ excitons measured without radiofrequency excitation and the splitting $\left(E_{\Uparrow \uparrow}^{k}-E_{\Uparrow \downarrow}^{k}\right)$ of the same excitons but measured after erasure of the nuclear polarization corresponding to the $k$ th isotope by the radiofrequency pulse. In the same way, the hole hyperfine shift is measured as $\Delta E_{\mathrm{h}}^{k}=\left(E_{\Uparrow \uparrow}-E_{\Downarrow \uparrow}\right)-\left(E_{\Uparrow \uparrow}^{k}-E_{\Downarrow \uparrow}^{k}\right)$.

The actual techniques for isotope-selective depolarization of nuclear spins depend on quantum-dot material. For example, in the case of GaAs/AlGaAs quantum dots, we take into account that both ${ }^{69} \mathrm{Ga}$ and ${ }^{71} \mathrm{Ga}$ isotopes have nearly equal chemical properties resulting in equal values of the relative hole hyperfine interaction constants $\gamma^{69} \mathrm{Ga}=\gamma^{71} \mathrm{Ga}=\gamma^{\mathrm{Ga}}$. Thus, measurement of $\gamma^{\mathrm{Ga}}$ can be accomplished by erasing both ${ }^{69} \mathrm{Ga}$ and ${ }^{71} \mathrm{Ga}$ polarization (which improves the measurement accuracy). In contrast, in InGaAs/GaAs quantum dots, ${ }^{115} \mathrm{In}$ and ${ }^{69} \mathrm{Ga}$ NMR spectra overlap owing to strain-induced quadrupole effects ${ }^{16,30}$, and $\gamma^{\text {In }}$ is extracted by calculating the hyperfine shifts of ${ }^{69} \mathrm{Ga}$ from the measured hyperfine shifts of ${ }^{71} \mathrm{Ga}$. Further details of the isotope-selective experimental techniques can be found in Supplementary Section S3. 
Theoretical model. First-principle calculation of the valence-band hyperfine coupling requires integration of the hyperfine Hamiltonian using explicit expressions for the Bloch wavefunctions. Each nucleus is coupled to a hole that spreads over many unit cells. However, it has been shown that the main effect arises from the short-range part of the dipole-dipole interaction ${ }^{11,15}$ (that is, coupling of the nuclear spin with the wavefunction within the same unit cell). This allows a simplified approach to be used: the Bloch functions of the valence-band maximum (corresponding to heavy-hole states) can be taken in the form $(-1 / \sqrt{2})(\mathcal{X}(\mathbf{r})+i \mathcal{Y}(\mathbf{r}))|\uparrow\rangle$ and $(1 / \sqrt{2})(\mathcal{X}(\mathbf{r})-i \mathcal{Y}(\mathbf{r}))|\downarrow\rangle$, where $|\uparrow\rangle,|\downarrow\rangle$ are spinors with corresponding spin projections on the $O z$ axis and $\mathcal{X}(\mathbf{r})=X(\theta, \phi) R(r), \mathcal{Y}(\mathbf{r})=Y(\theta, \phi) R(r)$ are orbitals that transform according to the $F_{2}$ representation of the $T_{d}$ point group relevant to bulk zinc-blende crystals (such as GaAs). Here, the $\mathcal{X}(\mathbf{r})$ and $\mathcal{Y}(\mathbf{r})$ orbitals are decomposed into a real radial part $R(r)$ and angular parts $X(\theta, \phi), Y(\theta, \phi)$. As a first approximation, the angular parts of the orbitals can be taken in the form $X_{p} \propto x / r, Y_{p} \propto y / r$ (corresponding to $p$-type states with orbital momentum $l=1$ ), and $R_{p}(r)$ can be approximated by hydrogenic radial functions ${ }^{10,11,15}$

To explain the opposite signs of the hole hyperfine constants observed experimentally we also need to take into account the contribution of the $d$-shell states $(l=2)$. To calculate the hyperfine constants (equation (3)) we assume that the heavy-hole orbitals can be taken as normalized linear combinations of the form $\mathcal{X}(\mathbf{r})=\alpha_{p} X_{p}(\theta, \phi) R_{p}(r)+\alpha_{d} X_{d}(\theta, \phi) R_{d}(r)$ and $\mathcal{Y}(\mathbf{r})=\alpha_{p} Y_{p}(\theta, \phi) R_{p}(r)+\alpha_{d} Y_{d}(\theta, \phi) R_{d}(r)$, where $\alpha_{l}$ are weighting coefficients $\left(\left|\alpha_{p}\right|^{2}+\left|\alpha_{d}\right|^{2}=1\right)$ and all orbitals $X_{l}$ corresponding to orbital momentum $l$ transform according to the same $F_{2}$ representation $(d$-shell states have the form $\left.X_{d} \propto y z / r^{2}, Y_{d} \propto x z / r^{2}\right)$. Further details of the theoretical model can be found in Supplementary Sections S4 and S5.

\section{Received 27 June 2012; accepted 9 November 2012;} published online 23 December 2012

\section{References}

1. Brunner, D. et al. A coherent single-hole spin in a semiconductor. Science $\mathbf{3 2 5}$, 70-72 (2009).

2. Heiss, D. et al. Observation of extremely slow hole spin relaxation in self-assembled quantum dots. Phys. Rev. B 76, 241306 (2007).

3. Greilich, A., Carter, S. G., Kim, D., Bracker, A. S. \& Gammon, D. Optical control of one and two hole spins in interacting quantum dots. Nature Photon. 5, 702-708 (2011).

4. De Greve, K. et al. Ultrafast coherent control and suppressed nuclear feedback of a single quantum dot hole qubit. Nature Phys. 7, 872-878 (2011).

5. Khaetskii, A. V., Loss, D. \& Glazman, L. Electron spin decoherence in quantum dots due to interaction with nuclei. Phys. Rev. Lett. 88, 186802 (2002).

6. Bluhm, H. et al. Dephasing time of GaAs electron-spin qubits coupled to a nuclear bath exceeding $200 \mu$ s. Nature Phys. 7, 109-113 (2011).

7. Eble, B. et al. Hole-nuclear spin interaction in quantum dots. Phys. Rev. Lett. 102, 146601 (2009).

8. Fallahi, P., Yilmaz, S. T. \& Imamoğlu, A. Measurement of a heavy-hole hyperfine interaction in InGaAs quantum dots using resonance fluorescence. Phys. Rev. Lett. 105, 257402 (2010).

9. Chekhovich, E. A., Krysa, A. B., Skolnick, M. S. \& Tartakovskii, A. I. Direct measurement of the hole-nuclear spin interaction in single InP/GaInP quantum dots using photoluminescence spectroscopy. Phys. Rev. Lett. 106, 027402 (2011).

10. Testelin, C., Bernardot, F., Eble, B. \& Chamarro, M. Hole-spin dephasing time associated with hyperfine interaction in quantum dots. Phys. Rev. B 79, 195440 (2009).

11. Fischer, J., Coish, W. A., Bulaev, D. V. \& Loss, D. Spin decoherence of a heavy hole coupled to nuclear spins in a quantum dot. Phys. Rev. B 78, 155329 (2008).

12. Boguslawski, P. \& Gorczyca, I. Atomic-orbital interpretation of electronic structure of III-V semiconductors: GaAs versus AlAs. Semicond. Sci. Technol. 9, 2169-2177 (1994).
13. Van de Walle, C. G. \& Blöchl, P. E. First-principles calculations of hyperfine parameters. Phys. Rev. B 47, 4244-4255 (1993).

14. Abragam, A. The Principles of Nuclear Magnetism (Oxford Univ. Press, 1961)

15. Gryncharova, E. I. \& Perel, V. I. Relaxation of nuclear spins interacting with holes in semiconductors. Sov. Phys. Semicond. 11, 997 (1977).

16. Chekhovich, E. A. et al. Structural analysis of strained quantum dots using nuclear magnetic resonance. Nature Nanotech. 7, 646-650 (2012).

17. Chekhovich, E. A., Krysa, A. B., Skolnick, M. S. \& Tartakovskii, A. I. Light-polarization-independent nuclear spin alignment in a quantum dot. Phys. Rev. B 83, 125318 (2011).

18. Bayer, M. et al. Fine structure of neutral and charged excitons in self-assembled In(Ga)As/(Al)GaAs quantum dots. Phys. Rev. B 65, 195315 (2002).

19. Poem, E. et al. Accessing the dark exciton with light. Nature Phys. 6, 993-997 (2010).

20. Jancu, J-M., Scholz, R., Beltram, F. \& Bassani, F. Empirical spds* tight-binding calculation for cubic semiconductors: General method and material parameters. Phys. Rev. B 57, 6493-6507 (1998).

21. Clementi, E. \& Raimondi, D. L. Atomic screening constants from scf functions. J. Chem. Phys. 38, 2686-2689 (1963).

22. Persson, C. \& Zunger, A. $s-d$ coupling in zinc-blende semiconductors. Phys. Rev. B 68, 073205 (2003).

23. Chadi, D. Angular momentum decomposition of $k=0$ Bloch functions in group IV and zincblende crystals. Solid State Commun. 20, 361-364 (1976).

24. Díaz, J. G. \& Bryant, G. W. Electronic and optical fine structure of GaAs nanocrystals: The role of $d$ orbitals in a tight-binding approach. Phys. Rev. B 73, 075329 (2006).

25. Di Carlo, A. Microscopic theory of nanostructured semiconductor devices: Beyond the envelope-function approximation. Semicond. Sci. Technol. 18, R1-R31 (2003).

26. Merz, J. L., Faulkner, R. A. \& Dean, P. J. Excitonic molecule bound to the isoelectronic nitrogen trap in GaP. Phys. Rev. 188, 1228-1239 (1969).

27. Steger, M. et al. Information storage for over $180 \mathrm{~s}$ using donor spins in a $28 \mathrm{Si}$ semiconductor vacuum. Science 336, 1280-1283 (2012).

28. Makhonin, M. N. et al. Fast control of nuclear spin polarization in an optically pumped single quantum dot. Nature Mater. 10, 844-848 (2011).

29. Gammon, D. et al. Nuclear spectroscopy in single quantum dots: Nanoscopic Raman scattering and nuclear magnetic resonance. Science 277, 85-88 (1997).

30. Flisinski, K. et al. Optically detected magnetic resonance at the quadrupolesplit nuclear states in (In,Ga)As/GaAs quantum dots. Phys. Rev. B 82, 081308 (2010).

\section{Acknowledgements}

The authors are grateful to M. Nestoklon, A. J. Ramsay, D. N. Krizhanovskii and M. Potemski for fruitful discussion and to D. Martrou for help with the GaAs sample growth. This work has been supported by EPSRC Programme Grants Nos EP/G001642/1 and EP/J007544/1, the Royal Society, ITN Spin-Optronics and ITN S³ NANO. M.M.G. was supported by the RFBR, Russian Federation President Grant NSh-5442.2012.2 and EU project SPANGL4Q.

\section{Author contributions}

A.B.K., M.H., P.S. and A.L. developed and grew the samples. E.A.C. and A.I.T. conceived the experiments. E.A.C. developed the techniques and carried out the experiments. E.A.C., M.M.G. and A.I.T. analysed the data. E.A.C., M.M.G., A.I.T. and M.S.S. wrote the manuscript with input from all authors.

\section{Additional information}

Supplementary information is available in the online version of the paper. Reprints and permissions information is available online at www.nature.com/reprints. Correspondence and requests for materials should be addressed to E.A.C. or A.I.T.

\section{Competing financial interests}

The authors declare no competing financial interests. 